\title{
Electrical Conductivity Study on Photo-induced Ionic Polymerization of $a$-Methylstyrene
}

\author{
Kanae HAYASHI \\ Osaka Laboratory, Japan Atomic Energy Research Institute, \\ 508 Mii Neyagawa, Osaka, Japan. \\ Masahiro IRIE and Koichiro HAYASHI \\ Faculty of Engineering, Hokkaido University, Sapporo, Japan. \\ (Received February 19, 1972)
}

KEY WORDS Cationic Polymerization / Charge-Transfer Interaction / Photopolymerization / Electrical Conductivity /

In the previous report ${ }^{1}$, photopolymerization of $\alpha$-methylstyrene in the presence of tetracyanobenzene was studied. The effect of additives and copolymerization with styrene indicated that the polymerization proceeds by a cationic mechanism. ${ }^{1} \alpha$-Methylstyrene and tetracyanobenzene form a weak electron donor-acceptor complex giving an absorption peak at $363 \mathrm{~nm}$ and the photo-excited complex is considered to initiate the polymerization. In this communication photoconductivity measurements have been carried out in the polymerization system to obtain further evidence of ionic polymerization.

The preparation of tetracyanobenzene and purification of the monomer and solvents were described before ${ }^{1}$. A xenon flash with a half width of $1.3 \mathrm{msec}$ was used as a light source. Photocurrent was measured by means of a Philbrik P2AU operational amplifier and a Iwasaki synchroscope (Model MS-5103B). The conductivity cell was of pyrex glass with stainless steel parallel plate electrodes of $15-\mathrm{mm}$ diameter situated at a distance of $18 \mathrm{~mm}$. The excitation wavelength was selected by Toshiba color filters. The light intensity was controlled by neutral filters. Measurements were carried out at $24^{\circ} \mathrm{C}$ and the observed current was always Ohmic.

Photoillumination at the wavelengths longer than $310 \mathrm{~nm}$ gives rise to a photocurrent in a methylene chloride solution containing $1.1 \mathrm{M}$ of $\alpha$-methylstryrene and $1 \times 10^{-3} \mathrm{M}$ of tetracyanobenzene, as shown by curve (a) in Figure 1,

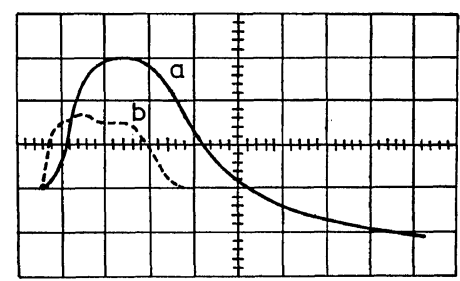

Figure 1. Photocurrent observed in methylene chloride solution containing $1.1 M$ of $\alpha$-methylstyrene and $1 \times 10^{-3} M$ of tetracyanobenzene by photo-illumination at wavelength longer than $310 \mathrm{~nm}$ at room temperature. Carve (b) shows the excitation lamp current; $500 \mu \mathrm{sec} / \mathrm{div}$ and $2 \mu \mathrm{A} /$ div. Applied voltage is $163 \mathrm{~V} / \mathrm{cm}$.

while no current was observed in the absence of $\alpha$-methylstyrene. Curve (b) in Figure 1 shows the excitation lamp current. The lifetime of ions is estimated to be several hundred microseconds or less. In this system, a decrease of the current to a lower level than that of the initial dark current was observed.

The photocurrent was observed so long as the excitation wavelength covered the chargetransfer band; it was not observed at all using illumination at a wavelength longer than $480 \mathrm{~nm}$. This indicates that ions are formed from photoexcited electron donor-acceptor complexes. In the polymerization study ${ }^{2}$, polymer was obtained using light which covers the wavelength of the charge-transfer band, but was not obtained using 
Electrical Conductivity Study on Photo-induced Ionic Polymerization of $\alpha$-Methylstyrene

light of wavelength longer than $450 \mathrm{~nm}$. The photocurrent as well as the polymer yield decreased markedly when oxygen was introduced into the sample. These correlations between free ion generation and polymerization indicate that both processes occur from a single precursor, photoexcited electron donor-acceptor complex.

For experimental convenience in clarifying the behaviors of free ions, photoconductivity measurement in $n$-amylalcohol containg $\alpha$-methylstyrene and tetracyanobenzene was also carried out, as shown in Figure 2 (a). Though in $n$ amylalcohol the propagation reaction hardly oc-

(a)

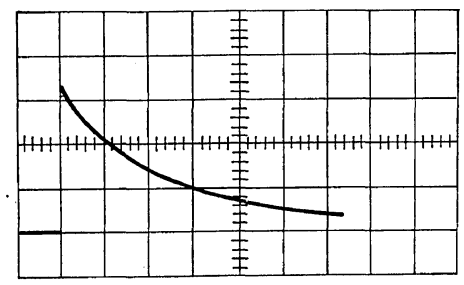

(b)

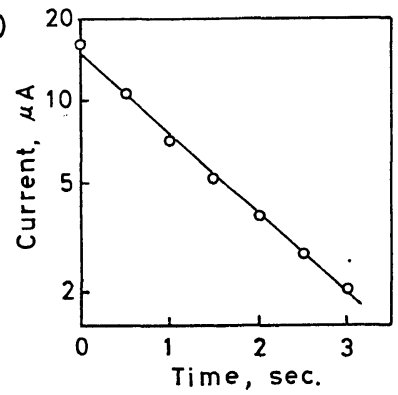

Figure 2. (a) Photocurrent observed in $n$-amylalcohol containig $1 \times 10^{-3} M$ of tetracyanobenzene and $7.6 \times 10^{-1} M$ of $\alpha$-methylstyrene after a flash at the wavelength longer than $310 \mathrm{~nm}$. Applied voltage is $163 \mathrm{~V} / \mathrm{cm}$. $5 \mu \mathrm{A} / \mathrm{div}$ and $500 \mathrm{msec} / \mathrm{div}$. (b) The logarithm plot of photocurrent. curs due to proton-transfer reaction, the experimental results obtained by photoconductivity measurement are thought to gives a clue to the initiation mechanism of the polymerization.

In this case also, photocurrent was observed only when the stimulating light covered the absorption range of the complex. The initial slope of the current was found to increase linearly with the increase in light intensity. Kinetic analysis of the current decay process, which is much slower than that in methylene chloride solution, showed a first-order disappearance of free ions, as shown in Figure 2(b). The rate constant obtained for the current decay is 0.65 $\mathrm{sec}^{-1}$, which is almost invariable with applied voltage. The first-order decay of free ions suggests that they are in equilibrium with an ion pair and disappearance of the ions are determined by the step of recombination of an ion pair as follows

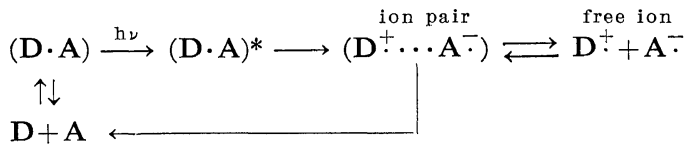

In this study, the existence of free ions, which are probably in an equilibrium with an ion pair, was confirmed in the photopolymerization system of $\alpha$-methylstyrene. Therefore in general, the polymerization is initiated with free-cationic and pair-cationic mechanisms coexistingly.

\section{REFERENCES}

1. M. Irie, S. Tomimoto, and K. Hayashi, J. Polym. Sci., Part B, 8, 585 (1970).

2. M. Irie, S. Tomimoto, and K. Hayashi, Abstracts, SPSJ 20th Annual Meeting, Tokyo, 1971, 24D20. 